\title{
Indices of the iterates of $\mathbb{R}^{3}$-homeomorphisms at fixed points which are isolated invariant sets
}

\author{
Patrice Le Calvez, Francisco R. Ruiz del Portal and José M. Salazar *
}

March 24, 2010

Dedicated to professor José M. Montesinos on the occasion of his 65th birthday.

\begin{abstract}
Let $U \subset \mathbb{R}^{3}$ be an open set and $f: U \rightarrow f(U) \subset \mathbb{R}^{3}$ be a homeomorphism. Let $p \in U$ be a fixed point. It is known that, if $\{p\}$ is not an isolated invariant set, the sequence of the fixed point indices of the iterates of $f$ at $p,\left(i\left(f^{n}, p\right)\right)_{n \geq 1}$, is, in general, unbounded. The main goal of this paper is to show that when $\{p\}$ is an isolated invariant set, the sequence $\left(i\left(f^{n}, p\right)\right)_{n \geq 1}$ is periodic. Conversely, we show that for any periodic sequence of integers $\left(I_{n}\right)_{n \geq 1}$ satisfying Dold's necessary congruences, there exists an orientation preserving homeomorphism such that $i\left(f^{n}, p\right)=I_{n}$ for every $n \geq 1$. Finally we also present an application to the study of the local structure of the stable/unstable sets at $p$.
\end{abstract}

\section{Introduction.}

Let $U \subset \mathbb{R}^{m}$ be an open set and $f: U \rightarrow f(U) \subset \mathbb{R}^{m}$ be a continuous map. Let $p \in U$ be a fixed point of $f$ that is an isolated fixed point of $f^{n}$ for every $n \geq 1$. Since Dold [4], it is known that the sequence $\left(i\left(f^{n}, p\right)\right)_{n \geq 1}$ of Lefschetz indices must satisfy some rules, called Dold's congruences. Shub and Sullivan proved that for $C^{1}$-maps the sequence is bounded. Chow, MalletParet and Yorke [3] gave bounds about the form of the sequence of indices in terms of the spectrum of the derivative $D f(p)$. Babenko and Bogatyi [1] proved that these bounds are sharp in dimension 2 and in a more recent paper [7] Graff and Nowak-Przygodzki have proved that for $n=3$ and $C^{1}$ maps, the sequence of fixed point indices follows one among exactly seven different periodic patterns.

\footnotetext{
${ }^{*}$ The authors have been supported by MICINN, MTM 2009-07030, the first author has also been supported by ANR (Symplexe, ANR-06-BLAN-0030-01) 2000 Mathematics Subject Classification: 37C25, 37B30, 54H25.

Keywords and phrases. Fixed point index, Conley index, entropy, filtration pairs.
} 
Suppose now that $f$ is a homeomorphism. For $m=2$, see [13], [14, [15] and [21], it is known that the sequence of indices $\left(i\left(f^{n}, p\right)\right)_{n \geq 1}$ is periodic with a very particular behavior. This sequence contains important dynamical information. For $m \geq 3$, the behavior is completely different from that of diffeomorphisms. If an isolated fixed point $p$ is not isolated as invariant set, the sequence of indices of the iterates in general is not bounded, even if the fixed point is Lyapunov stable. More precisely, in [22] it is proved that any sequence satisfying Dold's congruences is realized as the sequence of fixed point indices of the iterates of a $\mathbb{R}^{m}$-homeomorphism at an isolated and stable fixed point (see also [23]).

What happens if $p$ is an isolated invariant set ? There are examples, for $m=8$, where the sequence $\left(i\left(f^{n}, p\right)\right)_{n \geq 1}$ is unbounded (see [19]). The main result of the paper asserts that such a situation cannot occur if $m=3$, more precisely:

Theorem 1. Let $U \subset \mathbb{R}^{3}$ be an open set and $f: U \subset \mathbb{R}^{3} \rightarrow f(U) \subset \mathbb{R}^{3}$ be a homeomorphism. Let $p$ be a fixed point of $f$ such that $\{p\}$ is an isolated invariant set. Then the sequence $\left(i\left(f^{n}, p\right)\right)_{n \geq 1}$ is periodic.

From [1] (see also [11]), one knows that a bounded sequence that satisfies Dold's congruences is periodic. Therefore it would be sufficient to prove that the sequence is bounded in the main theorem. In fact we will prove directly the periodicity. If $f: M \rightarrow M$ is a continuous map on a $m$-dimensional compact manifold $M$, denote by $f_{* i}$ the induced map on the singular homology group $H_{i}(M, \mathbb{Q})$. A classical result of Manning [16] asserts that $\mathrm{h}_{\text {top }}(f) \geq \log \left(\operatorname{sr}\left(f_{* 1}\right)\right)$, where $\mathrm{h}_{\text {top }}(f)$ is the topological entropy of $f$ and $\operatorname{sr}\left(f_{* 1}\right)$ the spectral radius of $f_{* 1}$. As explained by Manning, this implies by Poincaré duality that if $f$ is a homeomorphism of a 3-dimensional compact manifold, one has $\mathrm{h}_{\text {top }}(f) \geq \sup _{0 \leq i \leq 3} \log \left(\operatorname{sr}\left(f_{* i}\right)\right)$. The inequality $\mathrm{h}_{\text {top }}(f) \geq \sup _{0 \leq i \leq n} \log \left(\operatorname{sr}\left(f_{i}^{*}\right)\right)$ is known to be true if $f$ is $C^{\infty}$ (see [28]) but fails to be true is $f$ is only continuous: what is constructed in [19] is an example of a homeomorphism on a 8-dimensional manifold where the previous inequality does not occur. As we will see later Manning's result will be the key-point of the proof of the main result. We will need to apply it, not on a manifold but on an absolute neighborhood retract ANR constructed via a filtration pair and we will need to use Lefschetz-Dold's formula to get a duality result. In fact we will prove the following more precise result.

Theorem 2. Let $U \subset \mathbb{R}^{3}$ be an open set and $f: U \subset \mathbb{R}^{3} \rightarrow f(U) \subset \mathbb{R}^{3}$ be a homeomorphism. Let $S$ be a connected compact isolated invariant set such that $h_{\mathrm{top}}\left(\left.f\right|_{S}\right)=0$. Then the sequence $\left(i\left(f^{n}, S\right)\right)_{n \geq 1}$ is periodic.

We will denote by $\operatorname{cl}(Y), \operatorname{int}(Y)$ and $\partial Y$ the closure, the interior and the frontier of a subset $Y$ of a topological space $X$. 


\subsection{Lefschetz index}

The reader is referred to [2, [4] and [18] for information about the Lefschetz index theory. Let us denote by $S^{m-1}$ the unit sphere of the euclidian space $\mathbb{R}^{m}$. Let $U \subset \mathbb{R}^{m}$ be a neighborhood of 0 and $f: U \rightarrow f(U)$ be a continuous map having an isolated fixed point at 0 . If $\varepsilon>0$ is small enough, the map

$$
z \mapsto \frac{\varepsilon z-f(\varepsilon z)}{\|\varepsilon z-f(\varepsilon z)\|}
$$

is well defined on $S^{m-1}$ and its degree does not depend on $\varepsilon$, it is the Lefschetz index $i(f, 0)$. For example, if $f$ is differentiable at 0 and 1 is not an eigenvalue of $D f(0)$, this number is equal to $(-1)^{l}$, where $l$ is the number of real eigenvalues larger than 1 . Recall the following facts in the case where $f$ is a homeomorphism :

- if $f$ preserves the orientation, then $i\left(f^{-1}, 0\right)=i(f, 0)$ if $m$ is even and $i\left(f^{-1}, 0\right)=-i(f, 0)$ if $m$ is odd;

- if $f$ reverses the orientation, then $i\left(f^{-1}, 0\right)=-i(f, 0)$ if $m$ is even and $i\left(f^{-1}, 0\right)=i(f, 0)$ if $m$ is odd.

Using charts one can define similarly the Lefschetz index of a continuous map on a manifold at an isolated fixed point $x$. Such a definition has been extended by Dold to the case where $f$ is defined on an ANR. Among the properties of the index, just recall that if $x$ is an attracting fixed point (i.e. there exists a compact neighborhood $V$ of $x$ satisfying $f(V) \subset V$ and $\left.\bigcap_{n \geq 0} f^{n}(V)=\{x\}\right)$ then one has $i(f, x)=1$. If $S \subset X$ is a compact invariant set (i.e. $f(S)=S$ ) and if there is a neighborhood $V$ of $S$ whose fixed points are all contained in $S$, one may define the index $i(f, S) \in \mathbb{Z}$. In the case where there are finitely many fixed points in $S$, it is equal to the sum of all Lefschetz indices of fixed points that are in $S$. A particular case is the case where $X$ is compact and $S=X$. The number $i(f, X)$ is called the Lefschetz number and denoted by $\Lambda(f)$. It is related to the action on the singular homology groups by the Lefschetz-Dold formula

$$
\Lambda(f)=\sum_{i=0}^{n}(-1)^{i} \operatorname{tr}\left(f_{* i}\right)
$$

where $f_{* i}$ is the morphism induced by $f$ on $H_{i}(X, \mathbb{Q})$ and $n$ the biggest integer such that $H_{i}(X, \mathbb{Q}) \neq 0$.

\subsection{Discrete Conley index, filtration pairs}

Let $U \subset M$ be an open set of a $m$-dimensional manifold $M$ and $f$ : $U \rightarrow f(U) \subset M$ be a homeomorphism. A compact invariant set $S$ is isolated with respect to $f$ if there exists a compact neighborhood $N$ of $S$ such 
that $\bigcap_{k \in \mathbb{Z}} f^{-k}(N)=S$. The neighborhood $N$ is called an isolating neighborhood of $S$. Let us say that $S$ is an attractor (resp. a repeller) if there exists a compact neighborhood $N$ of $S$ such that $\bigcap_{k \in \mathbb{N}} f^{k}(N)=S$ (resp. $\bigcap_{k \in \mathbb{N}} f^{-k}(N)=S$ ). Attractors and repellers are special classes of isolated invariant sets. An isolating block $N$ is a compact set such that $\operatorname{cl}(\operatorname{int}(N))=N$ and $f^{-1}(N) \cap N \cap f(N) \subset \operatorname{int}(N)$. Isolating blocks are a special class of isolating neighborhoods.

We consider the exit set of a set $N \subset U$ to be defined as

$$
N^{-}=\{x \in N: f(x) \notin \operatorname{int}(N)\} .
$$

Let $S$ be an isolated invariant set and suppose $L \subset N$ is a compact pair contained in $U$. The pair $(N, L)$ is called a filtration pair for $S$ (see Franks and Richeson paper [6]) provided $N$ and $L$ are each the closure of their interiors and

1) $\operatorname{cl}(N \backslash L)$ is an isolating neighborhood of $S$,

2) $L$ is a neighborhood of $N^{-}$in $N$,

3) $f(L) \cap \operatorname{cl}(N \backslash L)=\emptyset$.

We will recall in the next section how to construct a filtration pair $(N, L)$ that is simplicial. In that case, the quotient space $N_{L}$ obtained from the pair $(N, L)$ by an identification of $L$ to a point $[L]$ is an ANR. The properties of filtration pairs imply that this identification induces a continuous map $\bar{f}: N_{L} \rightarrow N_{L}$ that fixes $[L]$ and that sends each point $x \notin L$ to the projection in $N_{L}$ of $f(x)$. The fixed point $[L]$ is an attractor in the following strong sense : the map $\bar{f}$ is locally constant equal to $[L]$ on a neighborhood of $[L]$. In fact the dynamics of $\bar{f}$ is easy to understand : for every point $x$, either there exists an integer $n \geq 0$ such that $\bar{f}^{n}(x)=[L]$ or its $\omega$-limit set is included in $S$. One can apply the Lefschetz-Dold formula to each iterate of $\bar{f}$. Using the fact that $[L]$ is an attracting fixed point and that $H_{i}\left(N_{L}, \mathbb{Q}\right) \sim H_{i}(N, L, \mathbb{Q})$ (where $\sim$ means isomorphic), one gets

$$
\Lambda\left(\bar{f}^{n}\right)=\sum_{i=0}^{m}(-1)^{i} \operatorname{tr}\left(f_{* i}^{n}\right),
$$

where

$$
\Lambda\left(\bar{f}^{n}\right)=i\left(\bar{f}^{n},[L]\right)+i\left(\bar{f}^{n}, S\right)=1+i\left(f^{n}, S\right)
$$

The second aim of this article is to prove the following result:

Theorem 3. For any periodic sequence of integers $\left(I_{n}\right)_{n \geq 1}$ satisfying Dold's necessary congruences, there exists an orientation preserving homeomorphism of $\mathbb{R}^{3}$ that fixes $\{0\}$, that does not fixed any other compact set, such that $\{0\}$ admits an isolating block which is a topological ball and such that $i\left(f^{n}, 0\right)=I_{n}$ for every $n \geq 1$. 
The paper is organized as follows: the next section is devoted to the proof of the main theorem of this paper and to presenting its dynamical consequences. Section 3 is dedicated to Theorem 3.

\section{Proof of the Main Theorem.}

\subsection{Topological entropy}

The topological entropy is a numerical invariant related to the orbit growth. It represents the exponential growth rate for the number of orbit segments distinguishable with arbitrary fine precision. Let $f: X \rightarrow X$ be a continuous map with $X$ a compact metric space. We define an increasing sequence of metrics $d_{n}^{f}, n=1,2, \ldots$, with $d_{1}^{f}=d$,

$$
d_{n}^{f}(x, y)=\max _{0 \leq i \leq n-1} d\left(f^{i}(x), f^{i}(y)\right) .
$$

We denote the open balls

$$
B_{f}(x, \epsilon, n)=\left\{y \in X: d_{n}^{f}(x, y)<\epsilon\right\} .
$$

A set $E \subset X$ is said to be $(n, \epsilon)$-spanning if $X \subset \bigcup_{x \in E} B_{f}(x, \epsilon, n)$. If $S_{d}(f, \epsilon, n)$ is the minimal cardinality of an $(n, \epsilon)$-spanning set then we define the topological entropy of $f$ as

$$
h_{\text {top }}(f)=\lim _{\epsilon \rightarrow 0} \limsup _{n \rightarrow \infty} \frac{1}{n} \log S_{d}(f, \epsilon, n) .
$$

This definition does not depend on the metric $d$ but only on the topology of $X$.

It is known that the restriction of a map to the non-wandering set $\Omega(f)$ captures the entropy of the system. If $\Omega(f)$ is the (closed and invariant) set of points $x \in X$ such that for every open neighborhood $U$ of $x$ there is $n>0$ such that $f^{n}(U) \cap U \neq \emptyset$, then we have the following equality

$$
h_{\text {top }}(f)=h_{\text {top }}\left(\left.f\right|_{\Omega(f)}\right)
$$

due to Bowen (see [12], 20] or [27] for details).

Recall now the following result of Manning (Theorem 2 in [16]) which can be applied to spaces with nice local properties like finite CW-complexes of compact ANR's.

Theorem 4. If $X$ is a compact metric space with metric $d$ such that:

i) For every $\epsilon>0$ there exists $\delta>0$ such that if $d(x, y)<\delta$ then there exists a path $\sigma:[0,1] \rightarrow X$ from $x$ to $y$ with $\operatorname{diam}(\sigma([0,1])<\epsilon$.

ii) There exists $\epsilon_{0}>0$ such that any loop of diameter $<\epsilon_{0}$ is homotopically trivial in $X$.

Then for any continuous map $f: X \rightarrow X$

$$
h_{\text {top }}(f) \geq \log (|\lambda|)
$$

for every eigenvalue $\lambda$ of $f_{* 1}: H_{1}(X, \mathbb{Q}) \rightarrow H_{1}(X, \mathbb{Q})$. 


\subsection{Some algebraic lemmas}

Recall the classical following results :

Proposition 1. Let $A$ and $B$ two finitely generated $\mathbb{C}$-vector spaces and $u: A \rightarrow A$ and $v: B \rightarrow B$ two linear maps. If $\operatorname{tr}\left(u^{k}\right)=\operatorname{tr}\left(v^{k}\right)$ for every $k \geq 1$, then $u$ and $v$ have the same non-zero eigenvalues counted with their multiplicities

Proof. If $\left\{\lambda_{1}, \ldots, \lambda_{r}\right\}$ and $\left\{\mu_{1}, \ldots, \mu_{s}\right\}$ are the non-zero eigenvalues of $u$ and $v$ respectively, we have

$$
\sum_{k=1}^{\infty} \frac{\operatorname{tr}\left(u^{k}\right)}{k} Z^{k}=\sum_{k=1}^{\infty} \frac{\lambda_{1}^{k}+\cdots+\lambda_{r}^{k}}{k} Z^{k}=-\sum_{i=1}^{r} \log \left(1-\lambda_{i} Z\right) .
$$

The hypothesis gives us the polynomial equality

$$
\prod_{i=1}^{r}\left(1-\lambda_{i} Z\right)=\prod_{j=1}^{s}\left(1-\mu_{j} Z\right)
$$

which implies that $r=s$ and that for every $j$ there exists $i_{j}$ such that $\mu_{j}=\lambda_{i_{j}}$.

Proposition 2. Let $A$ be a finitely generated $\mathbb{C}$-vector space and $u: A \rightarrow A$ be a morphism which can be represented by a matrix $\left(a_{i j}\right)$ such that $a_{i j} \in \mathbb{Z}$ for all $i, j$. If all the eigenvalues of $u$ have modulus $\leq 1$, then all the non-zero eigenvalues are roots of unity.

Proof. The characteristic polynomial of $u$ may be written

$$
P_{u}(X)=X^{s} \prod_{1 \leq i \leq r}\left(X-\lambda_{i}\right)
$$

where $\lambda_{1}, \ldots, \lambda_{r}$ are the non-zero eigenvalues of $u$. The coefficients of $P_{u}$ being integers, one deduces that $\prod_{1 \leq i \leq r} \lambda_{i} \in \mathbb{Z}$. By hypothesis, this implies that each $\lambda_{i}$ satisfies $\left|\lambda_{i}\right|=1$ and may be written $\lambda_{i}=e^{2 i \pi \alpha_{i}}$, where $\alpha_{i} \in$ $\mathbb{R} / \mathbb{Z}$. One knows that every point of $\mathbb{R}^{r} / \mathbb{Z}^{r}$ is a recurrent point of the rotation

$$
\left(t_{1}, \ldots, t_{r}\right) \mapsto\left(t_{1}+\alpha_{1}, \ldots, t_{r}+\alpha_{r}\right) .
$$

One deduces that there exists $n>0$ such that every complex number $\lambda_{i}^{n}=$ $e^{2 i \pi n \alpha_{i}}$ is close to 1 . This implies that $\operatorname{tr}\left(u^{n}\right)=\lambda_{1}^{n}+\cdots+\lambda_{r}^{n}$ is close to $r$. But this number being an integer must be equal to $r$ and this implies that each $\lambda_{i}^{n}$ is equal to 1 . 


\subsection{Proof of Theorem 2 .}

Let us begin with a more detailed construction of filtration pairs (see also [6]).

Proposition 3. Let $U \subset \mathbb{R}^{m}$ be an open set and $f: U \rightarrow f(U) \subset \mathbb{R}^{m}$ be a homeomorphism. If $S$ is a connected invariant compact set isolated with respect to $f$, there exists a filtration pair $(N, L)$ for $S$ that is homeomorphic to a finite simplicial pair: the sets $N, L$ and $\operatorname{cl}(N \backslash L)$ are compact topological $m$-dimensional manifolds. More precisely:

1) if $S$ is neither an attractor, nor a repeller, one may suppose that $N$ is connected, that $L$ is not empty and that no bounded component of $\mathbb{R}^{m} \backslash L$ is included in $N$;

2) if $S$ is a repeller, one may suppose that $N$ is connected, that $L$ is not empty and that there exists a unique bounded component of $\mathbb{R}^{m} \backslash L$ that is included in $N$ and this component contains $S$;

3) if $S$ is an attractor, one may suppose that $N$ is connected and that $L$ is empty.

Proof. Suppose first that $S$ is neither an attractor, nor a repeller. One constructs first an isolating block, that means an isolating neighborhood $N$ of $S$ satisfying

$$
f(N) \cap N \cap f^{-1}(N) \subset \operatorname{int}(N),
$$

(see [6]) for example). Replacing $N$ by a small neighborhood that is a $m$ dimensional manifold, one may suppose that $N$ is itself a manifold. The connected component $N^{\prime}$ of $N$ that contains $S$ satisfies a similar equality because $\partial N^{\prime} \subset \partial N$. Replacing $N$ by $N^{\prime}$ one may suppose that $N$ is connected. The exit set $N^{-}$is not empty because $S$ is not an attractor. The inclusions $N^{-} \subset N$ and $f\left(N^{-}\right) \cap N \subset \partial N$ imply that

$$
f\left(N^{-}\right) \cap N \cap f^{-1}(N)=\emptyset .
$$

One can find a small neighborhood $L$ of $N^{-}$in $N$ satisfying

$$
f(L) \cap N \cap f^{-1}(N)=\emptyset,
$$

such that $(N, L)$ is a finite simplicial pair. Observe that $(N, L)$ is a filtration pair because $\operatorname{cl}(N \backslash L) \subset N \cap f^{-1}(N)$.

Write $W_{i}, 1 \leq i \leq r$ for the bounded connected components of $\mathbb{R}^{m} \backslash L$ that are included in $N$ and define $L^{\prime}=L \cup\left(\bigcup_{1 \leq i \leq r} W_{i}\right)$. To get the proposition, one must prove that $\left(N, L^{\prime}\right)$ is a filtration pair. As one knows that

$$
\operatorname{cl}\left(N \backslash L^{\prime}\right) \subset \operatorname{cl}(N \backslash L) \subset N \cap f^{-1}(N),
$$


it is sufficient to prove that for every $i \in\{1, \ldots, r\}$ one has $S \cap \operatorname{cl}\left(W_{i}\right)=\emptyset$ and $f\left(W_{i}\right) \cap N \cap f^{-1}(N)=\emptyset$. From

$$
f\left(\partial W_{i}\right) \cap \operatorname{cl}\left(W_{i}\right) \subset f(L) \cap \operatorname{cl}(N \backslash L)=\emptyset,
$$

one deduces that either $\operatorname{cl}\left(W_{i}\right) \subset f\left(W_{i}\right)$ or $\operatorname{cl}\left(W_{i}\right) \cap \operatorname{cl}\left(f\left(W_{i}\right)\right)=\emptyset$. In the first case the connected $S$ must be included in $W_{i}$ and equal to $\bigcap_{k \geq 0} f^{-k}\left(\operatorname{cl}\left(W_{i}\right)\right)$. It would be a repeller. So one knows that $\operatorname{cl}\left(W_{i}\right) \cap \operatorname{cl}\left(f\left(W_{i}\right)\right)=\emptyset$ and therefore that $S \cap \operatorname{cl}\left(W_{i}\right)=\emptyset$.

The inclusion $W_{i} \subset N \backslash N^{-}$implies that $f\left(W_{i}\right) \subset \operatorname{int}(N)$ and therefore that $f\left(\partial W_{i}\right) \subset N$. The inclusion $\partial W_{i} \subset L$ implies that

$$
f\left(\partial W_{i}\right) \cap f^{-1}(N) \subset f(L) \cap N \cap f^{-1}(N)=\emptyset .
$$

By connectedness of $f^{-1}(N)$, one deduces that either $f^{-1}(N) \subset f\left(W_{i}\right)$ or $f^{-1}(N) \cap \operatorname{cl}\left(f\left(W_{i}\right)\right)=\emptyset$. The first case does not occur because $S \subset f^{-1}(N)$ and $S \cap f\left(W_{i}\right)=\emptyset$. So we are in the second case and we deduce that $f\left(W_{i}\right) \cap N \cap f^{-1}(N)=\emptyset$.

In the case where $S$ is a repeller, one may find an isolating block $N$ such that $N \subset f(\operatorname{int}(N))$. We can suppose that it is a connected $m$-dimensional manifold and construct in a similar way a pair $(N, L)$. Observe that $\partial N \subset$ $N^{-} \subset L$. To get the proposition, keeping the same notations as before, we must prove that one of the $W_{i}$ contains $S$. In other words we must prove that the connected component of $\mathbb{R}^{m} \backslash L$ that contains $S$ is bounded and contained in $N$. It is a consequence of the fact that $W_{i}$ meets $N$ (it contains $S)$ but does not meet $\partial N$.

In the case where $S$ is an attractor one may find an isolating block $n$ such that $f(N) \subset \operatorname{int}(N)$. Here again one may suppose that $N$ is a connected compact $m$-dimensional manifold. The exit set is empty and $(N, \emptyset)$ satisfies the proposition.

Suppose that $(N, L)$ is a filtration pair that satisfies the previous proposition and write $\bar{f}: N_{L} \rightarrow N_{L}$ for the induced map.

Proposition 4. In the cases 1) and 2) one has $H_{0}\left(N_{L}, \mathbb{Q}\right) \sim \mathbb{Q}$, in the case 3) one has $H_{0}\left(N_{L}, \mathbb{Q}\right) \sim \mathbb{Q}^{2}$, in all cases one has $\bar{f}_{* 0}=\mathrm{Id}$.

In the cases 1) and 3) one has $H_{m}\left(N_{L}, \mathbb{Q}\right)=\{0\}$, in the case 2) one has $H_{m}\left(N_{L}, \mathbb{Q}\right) \sim \mathbb{Q}$. In this last case one has $\bar{f}_{* m}=$ Id if $f$ preserves the orientation and $\bar{f}_{* m}=-$ Id if $f$ reverses the orientation.

Proof. In cases 1 ) and 2), the set $N$ being connected and $L$ being non empty, one knows that $N_{L}$ is connected. Therefore $H_{0}\left(N_{L}, \mathbb{Q}\right)=\mathbb{Q}$ and obviously $\bar{f}_{* 0}=$ Id. In case 3$), N_{L}$ is no more connected because one add an isolated point. This point is fixed and the other component $N$ is sent into itself, therefore $H_{0}\left(N_{L}, \mathbb{Q}\right) \sim \mathbb{Q}^{2}$ and $\bar{f}_{* 0}=$ Id. 
Recall that $H_{m}\left(N_{L}, \mathbb{Q}\right)$ is equal to the relative homology group $H_{m}(N, L, \mathbb{Q})$. By Alexander's duality theorem (see [26], page 313) one knows that

$$
H_{m}(N, L, \mathbb{Q}) \sim H^{0}\left(\mathbb{R}^{m} \backslash L, \mathbb{R}^{m} \backslash N, \mathbb{Q}\right) .
$$

Therefore one must look at locally constant functions defined on $\mathbb{R}^{m} \backslash L$ that vanishes on $\mathbb{R}^{m} \backslash N$. Equivalently, one must look at bounded connected components of $\mathbb{R}^{m} \backslash L$ that are included in $N$. There is none in cases 1) and $3)$, there is exactly one in case 2$)$. In this case a generator of $H_{m}(N, L, \mathbb{Q})$ is given by $(\bar{W}, \partial W)$ where $W$ is the connected component of $\mathbb{R}^{m} \backslash L$ that contains $S$. Observe now that the pair $(f(\bar{W}), f(\partial W)) \subset(N, L)$ is homologous to $(\bar{W}, \partial W)$ if $f$ preserves the orientation and to $-(\bar{W}, \partial W)$ if $f$ reverses the orientation.

Let us prove now Theorem 2. We suppose that $m=3$ and that $h_{\text {top }}\left(\left.f\right|_{S}\right)=$ 0 . We choose a filtration pair $(N, L)$ satisfying Proposition 3. Suppose first that $S$ is neither an attractor, nor a repeller. From the two equalities

$$
\Lambda\left(\bar{f}^{n}\right)=\operatorname{tr}\left(\bar{f}_{*_{0}}^{n}\right)-\operatorname{tr}\left(\bar{f}_{* 1}^{n}\right)+\operatorname{tr}\left(\bar{f}_{* 2}^{n}\right)-\operatorname{tr}\left(\bar{f}_{* 3}^{n}\right)
$$

and

$$
\Lambda\left(\bar{f}^{n}\right)=i\left(\bar{f}^{n},[L]\right)+i\left(\bar{f}^{n}, S\right)=1+i\left(f^{n}, S\right),
$$

and from Proposition 4 we deduce that

$$
i\left(f^{n}, S\right)=-\operatorname{tr}\left(\bar{f}_{* 1}^{n}\right)+\operatorname{tr}\left(\bar{f}_{* 2}^{n}\right) .
$$

To prove that the sequence $\left(i\left(f^{n}, S\right)_{n \geq 1}\right.$ is bounded, it is sufficient to prove that the spectral radius of both maps $\bar{f}_{* 1}^{n}$ and $\bar{f}_{* 2}^{n}$ is bounded by 1 .

Since $H_{i}\left(N_{L}, \mathbb{Q}\right)=H_{i}\left(N_{L}, \mathbb{Z}\right) \otimes \mathbb{Q}$, Proposition 2 will imply that the eigenvalues are roots of unity, which will give us the periodicity condition. The fact that the spectral radius of the first map is bounded by 1 is a consequence of Manning's theorem. Indeed, the set of non wandering points $\Omega(\bar{f})$ is included in $\{[L]\} \cup S$ because $\Omega(\bar{f}) \backslash\{[L]\}$ is a closed set included in $N \backslash L$ and invariant by $f$. Therefore

$$
h_{\mathrm{top}}(\bar{f})=h_{\mathrm{top}}\left(\left.\bar{f}\right|_{\Omega(\bar{f})}\right)=h_{\mathrm{top}}\left(\left.f\right|_{S}\right)=0 .
$$

To prove a similar result for $\bar{f}_{*, 2}$ we will use the following duality argument. Let $\left(N, L^{\prime}\right)$ be a filtration pair for $f^{-1}$ satisfying Proposition 3 and let us consider the quotient space $N_{L^{\prime}}$ and the induced map $\overline{f^{-1}}: N_{L^{\prime}} \rightarrow N_{L^{\prime}}$.

The pointed union $M=N_{L} \vee N_{L^{\prime}}$ of $N_{L}$ and $N_{L^{\prime}}$ obtained by the identification of the points $[L]$ and $\left[L^{\prime}\right]$ to a point $*$ is a compact metric ANR and $*$ is an attractor of the induced map $\bar{g}=\bar{f} \vee \overline{f^{-1}}: M \rightarrow M$. Applying the Lefschetz-Dold formula, one gets 


$$
\Lambda\left(\bar{g}^{n}\right)=\operatorname{tr}\left(\bar{g}_{*_{0}}^{n}\right)-\operatorname{tr}\left(\bar{g}_{* 1}^{n}\right)+\operatorname{tr}\left(\bar{g}_{* 2}^{n}\right)-\operatorname{tr}\left(\bar{g}_{* 3}^{n}\right)
$$

where

$$
\Lambda\left(\bar{g}^{n}\right)=i\left(\bar{g}^{n}, *\right)+i\left(f^{n}, S\right)+i\left(f^{-n}, S\right) .
$$

We shall assume first that $f$ is orientation preserving. Then, $i\left(f^{n}, S\right)=$ $-i\left(f^{-n}, S\right)$ and it follows that $\Lambda\left(\bar{g}^{n}\right)=1$. On the other hand, one knows that $\operatorname{tr}\left(\bar{g}_{*_{0}}^{n}\right)=1$, that each space $H_{i}(M, \mathbb{Q}), 1 \leq i \leq 3$ may be written $H_{i}(M, \mathbb{Q})=$ $H_{i}\left(N_{L}, \mathbb{Q}\right) \oplus H_{i}\left(N_{L^{\prime}}^{\prime}, \mathbb{Q}\right)$ and that each map $\bar{g}_{* i}$ may be decomposed as $\bar{g}_{* i}=$ $\bar{f}_{* i} \oplus \bar{f}^{-1}{ }_{* i}$. One deduces that

$$
-\operatorname{tr}\left(\bar{g}_{* 1}^{n}\right)+\operatorname{tr}\left(\bar{g}_{* 2}^{n}\right)=0,
$$

for every $n \geq 0$. By Proposition 1, this implies that the non vanishing eigenvalues of $\bar{g}_{* 1}$ are equal to the non vanishing eigenvalues of $\bar{g}_{* 2}$, with the same multiplicities. The spectral radius of $\bar{g}_{* 1}$ being $\leq 1$ because $h_{\text {top }}(\bar{g})=0$, we have a similar result for $\bar{g}_{* 2}$ and therefore a similar result for $\bar{f}_{* 2}$.

In the case where $f$ is orientation reversing, we will get

$$
-\operatorname{tr}\left(\bar{g}_{* 1}^{n}\right)+\operatorname{tr}\left(\bar{g}_{* 2}^{n}\right)=0,
$$

for every even number $n$. But this will be sufficient to prove that the non vanishing eigenvalues of $\bar{g}_{* 1}$ are equal, up to the sign, to the non vanishing eigenvalues of $\bar{g}_{* 2}$.

Is $S$ is an attractor of $f$, then it is a repeller of $f^{-1}$. We construct filtration pairs $(N, L)$ and $\left(N^{\prime}, L^{\prime}\right)$ for $f$ and $f^{-1}$ that satisfy Proposition 3. If $f$ is orientation preserving, by looking at $\Lambda(\bar{f})$ and $\Lambda\left(\overline{f^{-1}}\right)$ we get

$$
\begin{aligned}
1+i\left(f^{n}, S\right) & =2-\operatorname{tr}\left(\bar{f}_{* 1}^{n}\right)+\operatorname{tr}\left(\bar{f}_{* 2}^{n}\right), \\
1+i\left(f^{-n}, S\right) & =1-\operatorname{tr}\left(\left(\bar{f}_{* 1}^{-1}\right)^{n}\right)+\operatorname{tr}\left(\left(\bar{f}_{* 2}^{-1}\right)^{n}\right)-1 .
\end{aligned}
$$

and

$$
1=\Lambda\left(\bar{g}^{n}\right)=2-\operatorname{tr}\left(\bar{g}_{* 1}^{n}\right)+\operatorname{tr}\left(\bar{g}_{* 2}^{n}\right)-1
$$

and we deduce that

$$
-\operatorname{tr}\left(\left(\bar{g}_{* 1}^{n}\right)+\operatorname{tr}\left(\left(\bar{g}_{* 2}^{n}\right)=0 .\right.\right.
$$

We can conclude as in the first case. If $f$ is orientation reversing we will get the equality for even integers and this permits us to conclude. The case where $S$ is a repeller is treated in the same way.

Observe that we have proven the following:

Corollary 1. Let $U \subset \mathbb{R}^{3}$ be an open set and $f: U \subset \mathbb{R}^{3} \rightarrow f(U) \subset \mathbb{R}^{3}$ be a homeomorphism. Let $p$ be a fixed point of $f$ such that $\{p\}$ is an isolated invariant set. 
- If $p$ is an attracting fixed point, the sequence $\left(i\left(f^{n}, p\right)\right)_{n \geq 1}$ is constant equal to 1 ;

- If $p$ is a repelling fixed point and $f$ preserves the orientation, the sequence $\left(i\left(f^{n}, p\right)\right)_{n \geq 1}$ is constant equal to -1 ;

- If $p$ is a repelling fixed point and $f$ reverses the orientation, one as $i\left(f^{n}, p\right)=(-1)^{n+1}$;

- if $p$ is neither an attracting fixed point, nor a repelling fixed point, there exist roots of unity $\lambda_{1}, \ldots, \lambda_{r}, \mu_{1}, \ldots, \mu_{s}$ such that

$$
i\left(f^{n}, p\right)=-\sum_{i=1}^{r} \lambda_{i}^{n}+\sum_{j=1}^{s} \mu_{j}^{n} .
$$

Remark 1. Let $U \subset \mathbb{R}^{3}$ be an open set, $f: U \subset \mathbb{R}^{3} \rightarrow f(U) \subset \mathbb{R}^{3}$ be an orientation preserving homeomorphism and $K \subset U$ be a continuum that is an isolated invariant set. Using the same ideas as above we have that there exist $\lambda_{1}, \ldots, \lambda_{r}, \mu_{1}, \ldots, \mu_{s}$ such that

$$
i\left(f^{n}, K\right)=-\sum_{i=1}^{r} \lambda_{i}^{n}+\sum_{j=1}^{s} \mu_{j}^{n} .
$$

Taking into account that $h_{\mathrm{top}}\left(\left.f\right|_{K}\right)=h_{\mathrm{top}}\left(\left.f^{-1}\right|_{K}\right)$ we have that

$$
\limsup _{n} \frac{\log \left|i\left(f^{n}, K\right)\right|}{n} \leq h_{\mathrm{top}}\left(\left.f\right|_{K}\right)
$$

\subsection{Local Stable/unstable sets.}

Let $U \subset \mathbb{R}^{3}$ be an open set and let $f: U \subset \mathbb{R}^{3} \rightarrow f(U) \subset \mathbb{R}^{3}$ be an orientation preserving homeomorphism. Let $p$ be a fixed point that is an isolated invariant set and that is neither an attractor nor a repeller. Let us consider a filtration pair $(N, L)$ and let us define the local unstable set of $p$ in $\operatorname{cl}(N \backslash L)$ :

$$
\Lambda_{\mathrm{cl}(N \backslash L)}^{-}(p)=\bigcap_{n \geq 0} f^{n}(\operatorname{cl}(N \backslash L)) .
$$

A point $x$ belongs to $\Lambda_{\mathrm{cl}(N \backslash L)}^{-}(p)$ if and only if the sequence $\left(f^{-n}(x)\right)_{n \geq 0}$ is well defined and takes its values in $\operatorname{cl}(N \backslash L)$. Obviously the set $\Lambda_{\mathrm{cl}(N \backslash L)}^{-}(p)$ is backward invariant, which implies that $\bigcap_{n \geq 0} f^{-n}\left(\Lambda_{\mathrm{cl}(N \backslash L)}^{-}(p)\right)$ is invariant. $\operatorname{Ascl}(N \backslash L)$ is an isolating neighborhood of $\{p\}$, the set $\bigcap_{n \geq 0} f^{-n}\left(\Lambda_{\operatorname{cl}(N \backslash L)}^{-}(p)\right)$ 
is reduced to the singleton $\{p\}$. Therefore, every $x \in \Lambda_{\operatorname{cl}(N \backslash L)}^{-}(p)$ satisfies

$$
\lim _{n \rightarrow \infty} f^{-n}(x)=p .
$$

We will prove the following:

Theorem 5. In the situation of the above paragraph, suppose in addition that $i\left(f^{q}, p\right)=r>0$, where $q$ is the period of the sequence $\left(i\left(f^{n}, p\right)\right)_{n \geq 1}$.

a) The second Alexander-Cech cohomology group with compact supports $\check{H}_{c}^{2}\left(\Lambda_{\mathrm{cl}(N \backslash L)}^{-}(p) \backslash L, \mathbb{Q}\right)$ contains at least $r$ copies of $\mathbb{Q}$.

b) If $N_{L}$ can be embedded in $\mathbb{R}^{3}$ then the image of $\left(\Lambda_{\mathrm{cl}(N \backslash L)}^{-}(p) \cup L\right) / L$ by this embedding decomposes $\mathbb{R}^{3}$ into at least $r+1$ components.

c) The set $\Lambda_{\operatorname{cl}(N \backslash L)}^{-}(p) \backslash L$ decomposes $\operatorname{int}(N) \backslash L$ into at least $r+1-$ $\operatorname{dim} H_{1}(\operatorname{cl}(N \backslash L), \mathbb{Q})$ components. In particular, if $\operatorname{cl}(N \backslash L)$ is a closed ball, $\Lambda_{\mathrm{cl}(N \backslash L)}^{-}(p) \backslash L$ decomposes $\operatorname{int}(N) \backslash L$ into at least $r+1$ components.

Proof. The proof will involve shape theory arguments. More precisely, the homology and cohomology theory that is a shape invariant. It is more appropriate, to study spaces that can have bad local behavior, than the singular homology theory. We recommend the book of Mardešić and Segal ([17]) for information about the theory of shape.

We keep the same notations as in the previous sections. The set

$$
\operatorname{Inv}^{-}\left(N_{L}, \bar{f}\right)=\bigcap_{n \geq 0} \bar{f}^{n}\left(N_{L}\right)
$$

is a continuum, being the intersection of a decreasing sequence of continua (recall that $N$ is connected and $L$ not empty). Observe that it is the image of $\Lambda_{\mathrm{cl}(N \backslash L)}^{-}(p)$ by the projection $\pi: N \rightarrow N_{L}$. Indeed if $x \in \Lambda_{\operatorname{cl}(N \backslash L)}^{-}(p)$ then for every $n \geq 0$, the point $x$ has a pre-image $f^{-n}(x)$ which is in $N$ and one has $\bar{f}^{n}\left(\pi\left(f^{-n}(x)\right)\right)=\pi(x)$. Conversely, suppose that $\xi \in\left(\operatorname{Inv}^{-}\left(N_{L}, \bar{f}\right)\right.$ is not equal to $[L]$ and equal to the projection of $x \in N \backslash L$. A point $\eta$ such that $\bar{f}^{n}(\eta)=\xi$ is not equal to $[L]$. Such a point is unique, it must be the projection of $f^{-n}(x)$. We have proven that

$$
\operatorname{Inv}^{-}\left(N_{L}, \bar{f}\right)=[L] \cup \pi\left(\Lambda_{\mathrm{cl}(N \backslash L)}^{-}(p)\right) .
$$

As we know that the set on the left is connected and both sets on the right are compact, we deduce that $[L] \in \pi\left(\Lambda_{\mathrm{cl}(N \backslash L)}^{-}(p)\right)$. In other words, $\operatorname{Inv}^{-}\left(N_{L}, \bar{f}\right)$ is Alexandroff's compactification of $\Lambda_{\mathrm{cl}(N \backslash L)}^{-}(p) \backslash L$.

Without loss of generality we can assume that $q=1$. In this case we have that all the eigenvalues of $\bar{f}_{* 1}$ and $\bar{f}_{* 2}$ are equal to 1 . Since the Lefschetz 
number can be computed also in terms of the cohomology groups the same statement holds for

$$
\bar{f}_{1}^{*}: H^{1}\left(N_{L}, \mathbb{Q}\right) \rightarrow H^{1}\left(N_{L}, \mathbb{Q}\right)
$$

and

$$
\bar{f}_{2}^{*}: H^{2}\left(N_{L}, \mathbb{Q}\right) \rightarrow H^{2}\left(N_{L}, \mathbb{Q}\right) .
$$

The set $\operatorname{Inv}^{-}\left(N_{L}, \bar{f}\right)$ is the inverse limit of the sequence

$$
\ldots N_{L} \stackrel{\bar{f}}{\rightarrow} N_{L} \stackrel{\bar{f}}{\rightarrow} N_{L} \stackrel{\bar{f}}{\rightarrow} N_{L} \ldots
$$

Since $i(f, p)=r>0$, there are at least $r$ eigenvalues of $\bar{f}_{2}^{*}$ that are equal to 1 . As a consequence, the (co)pro-group associated to the above inverse system

$$
\ldots H^{2}\left(N_{L}, \mathbb{Q}\right) \stackrel{\bar{f}_{2}^{*}}{\rightarrow} H^{2}\left(N_{L}, \mathbb{Q}\right) \stackrel{\bar{f}_{2}^{*}}{\rightarrow} H^{2}\left(N_{L}, \mathbb{Q}\right) \stackrel{\bar{f}_{2}^{*}}{\rightarrow} H^{2}\left(N_{L}, \mathbb{Q}\right) \ldots
$$

is nontrivial and the Alexander- $\check{C}$ ech cohomology groups

$\check{H}^{2}\left(\operatorname{Inv}^{-}\left(N_{L}, \bar{f}\right), \mathbb{Q}\right)=\check{H}^{2}\left(\left(\Lambda_{\mathrm{cl}(N \backslash L)}^{-}(p) \cup L\right) / L, \mathbb{Q}\right)=\check{H}^{2}\left(\Lambda_{\mathrm{cl}(N \backslash L)}^{-}(p) \cup L, L, \mathbb{Q}\right)$

contain at least $r$ copies of $\mathbb{Q}$.

As we know that

$$
\check{H}^{2}\left(\Lambda_{\mathrm{cl}(N \backslash L)}^{-}(p) \cup L, L, \mathbb{Q}\right)=\check{H}_{c}^{2}\left(\Lambda_{\mathrm{cl}(N \backslash L)}^{-}(p) \backslash L, \mathbb{Q}\right) .
$$

(see [25], page 321 , Lemma 11) we deduce that $\check{H}_{c}^{2}\left(\Lambda_{\mathrm{cl}(N \backslash L)}^{-}(p) \backslash L, \mathbb{Q}\right)$ contains at least $r$ copies of $\mathbb{Q}$. This proves a).

In order to check b) let us consider an embedding $\iota: N_{L} \rightarrow \mathbb{R}^{3}$. We know that

$$
\tilde{H}_{0}\left(\mathbb{R}^{3} \backslash \iota\left(\left(\Lambda_{\mathrm{cl}(N \backslash L)}^{-}(p) \cup L\right) / L\right), \mathbb{Q}\right)=\check{H}^{2}\left(\iota\left(\left(\Lambda_{\mathrm{cl}(N \backslash L)}^{-}(p) \cup L\right) / L\right), \mathbb{Q}\right)
$$

(see 25], page 296, Theorem 16). The fact that $\left.\check{H}^{2}\left(\left(\Lambda_{\mathrm{cl}(N \backslash L)}^{-}(p) \cup L\right) / L\right), \mathbb{Q}\right)$ contains $r$ copies of $\mathbb{Q}$ implies that $\iota\left(\left(\Lambda_{c l(N \backslash L)}^{-}(p) \cup L\right) / L\right)$ decomposes $\mathbb{R}^{3}$ into at least $r+1$ components.

Now we are going to prove c). Using Theorem 10 in page 342 of [25], we have that

$H_{1}\left(\operatorname{int}(N) \backslash L,(\operatorname{int}(N) \backslash L) \backslash\left(\Lambda_{\mathrm{cl}(N \backslash L)}^{-}(p) \backslash L\right), \mathbb{Q}\right)=\check{H}_{c}^{2}\left(\left(\Lambda_{\mathrm{cl}(N \backslash L)}^{-}(p) \backslash L\right), \mathbb{Q}\right)$.

Then, from a) and the exactness of the homology sequence of the pair $\left(\operatorname{int}(N) \backslash L,(\operatorname{int}(N) \backslash L) \backslash\left(\Lambda_{\mathrm{cl}(N \backslash L)}^{-}(p) \backslash L\right)\right)$, if follows that

$\left.\operatorname{dim} H_{0}(\operatorname{int}(N) \backslash L) \backslash\left(\Lambda_{\mathrm{cl}(N \backslash L)}^{-}(p) \backslash L\right), \mathbb{Q}\right) \geq r+1-\operatorname{dim} H_{1}(\operatorname{cl}(N \backslash L), \mathbb{Q})$. 
Remark 2. Note that using shape theory arguments it is easy to check that the covering dimension $\operatorname{dim}\left(\operatorname{Inv}^{-}\left(N_{L}, \bar{f}\right)\right) \geq 2$. Indeed, pro $H_{2}\left(\operatorname{Inv}^{-}\left(N_{L}, \bar{f}\right), \mathbb{Q}\right)$ can be also computed in terms of the $\check{C}$ ech expansion of $\operatorname{Inv}^{-}\left(N_{L}, \bar{f}\right)$, it follows that there is a cofinal family of open coverings of $\operatorname{Inv}^{-}\left(N_{L}, \bar{f}\right)$ whose nerves are polyhedra of dimension $\geq 2$. Then, there is a cofinal family of open coverings of $\operatorname{Inv}^{-}\left(N_{L}, \bar{f}\right)$ of order $\geq 3$. Then, the covering dimension $\operatorname{dim}\left(\operatorname{Inv}^{-}\left(N_{L}, \bar{f}\right)\right) \geq 2$.

Remark 3. If in the above theorem $f$ is orientation preserving and $i\left(f^{q}, p\right)=r<0$ we obtain a similar result for the local unstable set of $\{p\}$ associated to $f^{-1}$.

\section{Proof of Theorem 3.}

We have proved that the sequence $\left(i\left(f^{n}, p\right)\right)_{n \geq 1}$ is periodic. Let us answer the converse question, i.e., for any periodic sequence of integers $I=\left(I_{n}\right)_{n \geq 1}$, which satisfies Dold's necessary congruences, there exists a homeomorphism with $i\left(f^{n}, p\right)=I_{n}$ for every $n \geq 1$. Moreover, we can construct the homeomorphism $f: \mathbb{R}^{3} \rightarrow \mathbb{R}^{3}$ to be orientation preserving with no compact invariant sets but $\{p\}$.

There are different ways to define Dold's congruences. The simplest one uses the normalized periodic sequences $\sigma^{k}=\left(\sigma_{n}^{k}\right)_{n \geq 1}$, where for each $k \geq 1$, one has

$$
\sigma_{n}^{k}= \begin{cases}k & \text { if } n \in k \mathbb{N} \\ 0 & \text { if } n \notin k \mathbb{N}\end{cases}
$$

Any sequence $I=\left(I_{n}\right)_{n \geq 1}$ may be written formally $I=\sum_{k \geq 1} a_{k} \sigma^{k}$ in an unique way. It satisfies Dold's congruences if and only if the $a_{k}$ are integers. As proved by Dold ([4]), a sequence of integers $I=\left(I_{n}\right)_{n \geq 1}$ satisfies Dold's congruences if and only if there exists an ENR $X$, a continuous map $f: X \rightarrow$ $X$ and an open subset $U \subset X$ such that for every $n \geq 1$, the set $\operatorname{Fix}\left(f^{n}\right) \cap U_{n}$ is compact and $I_{n}=i_{X}\left(f^{n}, U_{n}\right)$, the sequence $\left(U_{n}\right)_{n \geq 1}$ being inductively defined by $U_{1}=U$ and $U_{n}=f^{-1}\left(U_{n-1}\right) \cap U$. As observed by Babenko and Bogatyi (see [1] or [11]), a bounded sequence that satisfies Dold's congruences must be periodic. That means that the sum $I=\sum_{k \geq 1} a_{k} \sigma^{k}$ is finite.

To prove Theorem 3 we will find a map in the class of radial homeomorphisms. The 3-dimensional sphere is nothing but the end compactification of $S^{2} \times \mathbb{R}$ where one adds to $S^{2} \times \mathbb{R}$ the lower end $e^{-}$, adherent to $S^{2} \times(-\infty, 0]$, and the upper end $e^{+}$, adherent to $S^{2} \times[0,+\infty)$. Let us fix a homeomorphism $g$ of $S^{2}$ and a continuous map $\varphi: S^{2} \rightarrow \mathbb{R}$. The skew product

$$
f_{g, \varphi}:(x, y) \mapsto(g(x), y+\varphi(x))
$$

induces on $S^{3}$ a homeomorphism fixing the two ends. If one wants $\left\{e^{-}\right\}$to be locally maximal it is sufficient to suppose that the following property $(\mathrm{P})$ is satisfied: 
(P) for every $x \in S^{2}, \varphi(x) \geq 0 \Rightarrow \varphi(g(x))>0$.

Indeed the property $(\mathrm{P})$ implies that there exists $\varepsilon>0$ such that for every $x \in S^{2}, \varphi(x) \geq-\varepsilon \Rightarrow \varphi(g(x)) \geq \varepsilon$. Writing

$$
f_{g, \varphi}^{-1}:(x, y) \mapsto\left(g^{-1}(x), y-\varphi\left(g^{-1}(x)\right)\right),
$$

one deduces that every orbit $\left(x_{k}, y_{k}\right)_{k \in \mathbb{Z}}$ of $f_{g, \varphi}$ satisfies at least one of the following properties:

- $y_{k+1}-y_{k} \geq \varepsilon$ for every $k \geq 0$

or

- $y_{k-1}-y_{k} \geq \varepsilon$ for every $k \leq 0$.

This implies that either $\lim _{k \rightarrow+\infty} y_{k}=+\infty$ or $\lim _{k \rightarrow-\infty} y_{k}=+\infty$. This also implies that each ball $\left(S^{2} \times(-\infty, r]\right) \cup\left\{e^{-}\right\}$is an isolating block.

Write $X_{0}=\varphi^{-1}([0,+\infty))$. The following points are easy to state:

- the exit set of $N=\left(S^{2} \times(-\infty, 0]\right) \cup\left\{e^{-}\right\}$is $N^{-}=\left\{(x, y) \mid x \in X_{0},-\varphi(x) \leq\right.$ $y \leq 0\}$;

- the unstable set of $e^{-}$is $X^{-} \times \mathbb{R} \cup\left\{e^{-}\right\}$where $X^{-}=\bigcap_{k>0} g^{k}\left(X_{0}\right)$, it is the infinite cone over $X^{-}$;

- the stable set of $e^{-}$is $X^{+} \times \mathbb{R}$ where $X^{+}=\bigcap_{k \leq 0} g^{k}\left(S^{2} \backslash X_{0}\right)$;

- one obtains a filtration pair $(N, L)$ writing $L=\{(x, y) \mid x \in X,-\psi(x) \leq$ $y \leq 0\}$ where $X \subset S$ is a subsurface with boundary, neighborhood of $X_{0}$ satisfying $g(X) \subset \operatorname{int}\left(X_{0}\right)$, and $\psi: X \rightarrow[0, \infty)$ a continuous function vanishing exactly on $\partial X$ and satisfying $\psi(g(x))>\psi(x)-\varphi(x)>0$ for every $x \in X$.

One can compute the Lefschetz index $i\left(f_{g, \varphi}^{n}, e^{-}\right)$by looking at the induced maps on the spaces $H_{i}(N, L, \mathbb{Q})$. We will give an alternative way, supposing for convenience that $X_{0}$ itself is a subsurface with boundary.

Proposition 5. For every $n \geq 1$, one has $i\left(f_{g, \varphi}^{n}, e^{-}\right)=1-\Lambda\left(\left(\left.g\right|_{X_{0}}\right)^{n}\right)$, where

$$
\Lambda\left(\left(\left.g\right|_{X_{0}}\right)^{n}\right)=\operatorname{tr}\left(\left(\left.g\right|_{X_{0}}\right)_{* 0}\right)^{n}-\operatorname{tr}\left(\left(\left.g\right|_{X_{0}}\right)_{* 1}\right)^{n}
$$

is the Lefschetz number of the $n$-th iterate of $\left.g\right|_{X_{0}}: X_{0} \rightarrow X_{0}$.

Proof. Write $N^{+}=\left\{(x, y) \mid x \in X_{0}, 0 \leq y \leq \varphi(x)\right\}$ and observe that $N^{\prime}=$ $N \cup N^{+}$is a 3 -dimensional sphere. One constructs a continuous map $\bar{f}_{g, \varphi}$ : $N^{\prime} \rightarrow N^{\prime}$ satisfying

$$
\bar{f}_{g, \varphi}(x, y)= \begin{cases}(g(x), 0) & \text { if }(x, y) \in N^{-} \cup N^{+} \\ f_{g, \varphi}(x, y) & \text { if }(x, y) \notin N^{-} \cup N^{+}\end{cases}
$$

Observe that both sets $N^{\prime}$ and $N^{-} \cup N^{+}$are attracting sets of $\bar{f}_{g, \varphi}$ and that $r:(x, y) \mapsto(x, 0)$ is a strong deformation retraction from $N^{-} \cup N^{+}$to 
$X_{0} \times\{0\}$ that satisfies $r \circ \bar{f}_{g, \varphi}=\left.g\right|_{X_{0}} \circ r$. This implies that

$$
\begin{aligned}
1 & =\Lambda\left(\left(\bar{f}_{g, \varphi}\right)^{n}\right) \\
& =i\left(f_{g, \varphi}^{n}, e^{-}\right)+\Lambda\left(\left(\left.\bar{f}_{g, \varphi}\right|_{N \cup N^{\prime}}\right)^{n}\right) \\
& =i\left(f_{g, \varphi}^{n}, e^{-}\right)+\Lambda\left(\left(\left.g\right|_{X_{0}}\right)^{n}\right) .
\end{aligned}
$$

Now we can prove Theorem 3. Let $I=\left(1+a_{1}\right) \sigma^{1}+\sum_{2 \leq k \leq k_{0}} a_{k} \sigma^{k}$ be a periodic sequence that satisfies Dold's congruences. Write

$$
A^{-}=\left\{k \geq 1 \mid a_{k}<0\right\}, A^{+}=\left\{k \geq 1 \mid a_{k}>0\right\},
$$

and define

$$
a^{-}=\sum_{k \in A^{-}} a_{k}, a^{+}=\sum_{k \in A^{+}} a_{k} .
$$

Choose $-a^{-}$closed disks $\left(Z_{i}^{k}\right)_{k \in A^{-}, 1 \leq i \leq-a_{k}}$ and $a^{+}$closed annuli $\left(Z_{i}^{k}\right)_{k \in A^{+}, 1 \leq i \leq a_{k}}$ on $S^{2}$, all pairwise disjoint. One can easily construct an orientation preserving homeomorphism $g$ of $S^{2}$ that satisfies the following properties:

- for each $k \in A^{-}$and each $i \in\left\{1, \ldots,-a_{k}\right\}$ there exist $k$ disjoint closed disks $D_{i, j}^{k}, j \in \mathbb{Z} / k \mathbb{Z}$, in the interior of $Z_{i}^{k}$ such that $g\left(D_{i, j}^{k}\right) \subset \operatorname{int}\left(D_{i, j+1}^{k}\right)$ and such that the maximal invariant set contained in $X_{i}^{k}=\bigcup_{j \in \mathbb{Z} / k \mathbb{Z}} D_{i, j}^{k}$ is a periodic orbit of period $k$;

- for each $k \in A^{+}$and each $i \in\left\{1, \ldots, a_{k}\right\}$ there exist $k$ disjoint closed disks $D_{i, j}^{k}, j \in \mathbb{Z} / k \mathbb{Z}$, in the interior of $Z_{i}^{k}$ such that $g^{-1}\left(D_{i, j}^{k}\right) \subset \operatorname{int}\left(D_{i, j+1}^{k}\right)$, such that $X_{i}^{k}=Z_{i}^{k} \backslash \bigcup_{j \in \mathbb{Z} /(k) \mathbb{Z}} \operatorname{int}\left(D_{i, j}^{k}\right)$ is an attracting set of $g$ and such that the maximal invariant set contained in $X_{i}^{k}$ is a wedge of $k+1$ circles.

Choose now a continuous function $\varphi: S^{2} \rightarrow \mathbb{R}$ that is positive in the interior of $X_{0}=\bigcup_{1 \leq k \leq k_{0}, 1 \leq i \leq\left|a_{k}\right|} X_{i}^{k}$ and negative outside $X_{0}$. Observe that

$$
\Lambda\left(\left(\left.g\right|_{X_{i}^{k}}\right)^{n}\right)= \begin{cases}k & \text { if } n \in k \mathbb{N} \\ 0 & \text { if } n \notin k \mathbb{N}\end{cases}
$$

if $k \in A^{-}$, and

$$
\Lambda\left(\left(\left.g\right|_{X_{i}^{k}}\right)^{n}\right)= \begin{cases}-k & \text { if } n \in k \mathbb{N}, \\ 0 & \text { if } n \notin k \mathbb{N}\end{cases}
$$

if $k \in A^{+}$. By Proposition 5, one deduces that the sequence $J=\left(i\left(f_{g, \varphi}^{n}, e^{-}\right)\right)_{n \geq 1}$ satisfies

$$
\begin{aligned}
J & =\sigma^{1}-\sum_{k \in A^{-}} \sum_{1 \leq i \leq-a_{k}} \sigma^{k}-\sum_{k \in A^{+}} \sum_{1 \leq i \leq a_{k}}\left(-\sigma^{k}\right) \\
& =\sigma^{1}+\sum_{k \in A^{-}} a_{k} \sigma^{k}+\sum_{k \in A^{+}} a_{k} \sigma^{k} \\
& =I
\end{aligned}
$$


Remark 4. Of course, a given sequence $I$ corresponds to infinitely many such constructions. In the construction above, the sequence $I^{0}=\sigma^{1}$ corresponds to a map $f_{g, \varphi}$ such that $\varphi<0$ (the ball $N$ being an attracting set), the sequence $I^{1}=-\sigma^{1}$ to a map $f_{g, \varphi}$ such that $X_{0}$ is the union of two attracting disks (an explicit example being given by the hyperbolic linear map $\left.l:\left(x_{1}, x_{2}, x_{3}\right) \mapsto\left(x_{1} / 2, x_{2} / 2,2 x_{3}\right)\right)$, the sequence $I^{2}=\sigma^{1}-\sigma^{2}$ to a map $f_{g, \varphi}$ such that $X_{0}$ is the union of two permuting disks (an explicit example being given by $\left.l^{\prime}:\left(x_{1}, x_{2}, x_{3}\right) \mapsto\left(x_{1} / 2, x_{1} / 2,-2 x_{3}\right)\right)$. Observe that $I^{0}$ also corresponds to a map $f_{g, \varphi}$ such that $X_{0}$ is an attracting annulus (an explicit example being given by $l^{-1}$ ) and that $I^{1}$ also corresponds to a map $f_{g, \varphi}$ such that $\varphi>0$ (the ball $N$ being a repelling set).

Remark 5. Applying the previous proposition to $f_{g, \varphi}^{-1}:(x, y) \mapsto$ $\left(g^{-1}(x), y-\varphi\left(g^{-1}(x)\right)\right)$, one knows that $i\left(f_{g, \varphi}^{-n}, e^{-}\right)=1-\Lambda\left(\left(\left.g^{-1}\right|_{S^{2} \backslash g\left(X_{0}\right.}\right)^{n}\right)$ for every $n \geq 1$. As we are on a 2-dimensional sphere and as we know that $g\left(X_{0}\right) \subset X_{0}$ we deduce that

$$
\Lambda\left(\left(\left.g^{-1}\right|_{S^{2} \backslash g\left(X_{0}\right)}\right)^{n}\right)=i\left(g^{-n}, X^{+}\right)=i\left(g^{n}, X^{+}\right),
$$

which implies that

$$
i\left(f_{g, \varphi}^{-n}, e^{-}\right)+\left(f_{g, \varphi}^{n}, e^{-}\right)=2-i\left(g^{n}, X^{-}\right)-i\left(g^{n}, X^{+}\right)=0
$$

by the Poincaré-Hopf formula.

Remark 6. All the constructions above may be done in higher dimension. Radial homeomorphisms may be constructed similarly and Proposition 5 may be generalized. Replacing all the disks by balls and the annuli by filled tori in the construction above, one gets a generalization of Theorem 3 in any dimension. These constructions permit us to understand why Theorem 1 is not true in higher dimension. One can construct a diffeomorphism $g$ on $S^{3}$ satisfying the following: there exists an embedded torus $T$ cutting the sphere in two solid tori $T^{-}$and $T^{+}$such that both maps $g: T^{+} \rightarrow T^{+}$and $g^{-1}: T^{-} \rightarrow T^{-}$are solenoidal maps of degree $m$, that means conjugate to a mapping

$$
(\theta, z) \mapsto\left(\theta^{m}, \frac{1}{2} \theta+\varepsilon z \theta^{k-m+1}\right)
$$

defined on the filled torus $\{\theta \in \mathbb{C}|| \theta \mid=1\} \times\{z \in \mathbb{C}|| z \mid \leq 1\}$ (see for example [10]). Let us consider a function $\varphi: S^{3} \rightarrow \mathbb{R}$ positive (resp. negative) on the interior of $T^{+}$(resp. $T^{-}$). The unstable manifold of $e^{-}$is an infinite cone over the solenoid $\bigcap_{n \geq 0} g^{n}\left(T^{+}\right)$and one knows that for every $n \geq 1$

$$
i\left(f_{g, \varphi}^{n}, e^{-}\right)=1-L\left(\left(\left.g\right|_{T^{+}}\right)^{n}\right)=1-\left(1-m^{n}\right)=m^{n}
$$

which implies that $\lim _{n \rightarrow+\infty} i\left(f_{g, \varphi}^{n}, e^{-}\right)=+\infty$. 
The authors would like to thank Federico Rodríguez Hertz, María Alejandra Rodríguez Hertz and Raúl Ures for indicating us a mistake in the construction of a previous example.

\section{References}

[1] I.K. Babenko, S.A. Bogatyi, The behavior of the index of periodic points under iterations of a mapping, Math. USSR Izvestiya, 38 (1992), 1-26.

[2] R.F. Brown, The Lefschetz fixed point theorem, Scott Foreman Co. Glenview Illinois, London (1971).

[3] S.N. Chow, J. Mallet-Paret, J.A. Yorke, A periodic orbit index which is a bifurcation invariant, Geometric Dynamics (Rio de Janeiro, 1981). Springer Lecture Notes in Math., 1007. Berlin 1983, 109-131.

[4] A. Dold, Fixed point indices of iterated maps, Invent. Math., 74, (1983), 419-435.

[5] J. Franks, The Conley index and non-existence of minimal homeomorphisms, Illinois Journal of Math. 43 (1999), 3, 457-464.

[6] J. Franks, D. Richeson, Shift equivalence and the Conley index, Trans. Amer. Math. Soc. 352, 7 (2000), 3305-3322.

[7] G. Graff, P. Nowak-Przygodzki, Fixed point indices of iterations of $C^{1}$ maps in $\mathbb{R}^{3}$, Discrete Contin. Dyn. Syst. 16 (2006), 4, 843-856.

[8] M. Handel, There are no minimal homeomorpisms of the multipunctured plane, Ergodic Theory Dynam. Systems, 12 (1992), 75-83.

[9] S.T. Hu, Theory of retracts, Wayne State University Press, 1965.

[10] J. H. Hubbard, R. W. Oberste-Vorth, Linked Solenoid Mappings and the Non-Transversality Locus Invariant, Indiana Univ. Math. J. , 50 (2001), 1, 553-566.

[11] J. Jezierski, W. Marzantowicz, Homotopy Methods in Topological Fixed and Periodic Points Theory, Springer, 2005.

[12] A. Katok, B. Hasselblatt, Introduction to the Modern Theory of Dynamical Systems, Cambridge University Press, Cambridge (1995).

[13] P. Le Calvez, J.C. Yoccoz, Un theoréme d'indice pour les homéomorphismes du plan au voisinage d'un point fixe, Annals of Math. 146 (1997), 241-293. 
[14] P. Le Calvez, J.C. Yoccoz, Suite des indices de Lefschetz des itérés pour un domaine de Jordan qui est un bloc isolant, Unpublished.

[15] P. Le Calvez, Dynamique des homéomorphismes du plan au voisinage d'un point fixe. Ann. Sci. Éc. Norm. Supér. (4) 36 (2003), 1, 139-171.

[16] A. Manning, Topological entropy and the first homology group, Dynamical Systems - Warwick 1974, Lecture Notes in Math. 468. Springer Verlag 1975, 185-190.

[17] S. Mardešić, J. Segal, Shape theory, North-Holland, Amsterdam, 1982.

[18] R.D. Nussbaum, The fixed point index and some applications, Séminaire de Mathématiques supérieures, Les Presses de L'Université de Montréal, 1985.

[19] C. Pugh, On the entropy conjecture, Dynamical Systems - Warwick 1974, Lecture Notes in Math. 468. Springer Verlag 1975, 257-261.

[20] C. Robinson, Dynamical systems. Stability, symbolic dynamics, and chaos, Studies in Advanced Mathematics. CRC Press, Boca Raton, FL, 1995.

[21] F.R. Ruiz del Portal, J.M. Salazar, Fixed point index of iterations of local homeomorphisms of the plane: a Conley-index approach, Topology 41 (2002), 1199-1212.

[22] F. R. Ruiz del Portal, J. M. Salazar, Realization of all Dold's congruences with stability, J. Diff. Equations (2010), doi:10.1016/j.jde.2010.03.006.

[23] F. R. Ruiz del Portal, J. M. Salazar, Indices of the iterates of $\mathbb{R}^{3}$ homeomorphisms at Lyapunov stable fixed points, Journal of Diff. Equations, 244 (2008), 1141-1156.

[24] M. Shub and D. Sullivan, A remark on the Lefschetz fixed point formula for differentiable maps, Topology, 13 (1974), 189-191.

[25] E. Spanier, Algebraic Topology, McGraw-Hill series in Higher Mathematics 1966.

[26] R. M. Switzer, Algebraic Topology - Homology and Homotopy, Die Grundlehren der math. Wissenschaften, 212. Springer Verlag, 1975.

[27] P. Walters, An Introduction to Ergodic Theory, Springer Verlag, 1982.

[28] Y. Yomdin, Volume growth and entropy, Israel J. Math., 57 (1987), 285-300. 
Patrice Le Calvez

Institut de Mathématiques de Jussieu (UMR 7586 CNRS), UPMC 175 rue du Chevaleret 75013 Paris, France.

E-mail: lecalvez@math.jussieu.fr

Francisco R. Ruiz del Portal

Departamento de Geometría y Topología, Facultad de CC.Matemáticas, Universidad Complutense de Madrid, Madrid 28040, Spain.

E-mail: R_Portal@mat.ucm.es

José Manuel Salazar.

Departamento de Matemáticas. Universidad de Alcalá. Alcalá de Henares. Madrid 28871, Spain.

E-mail: josem.salazar@uah.es 